\title{
Effects of the sweep rate of the applied field on the agglomeration of magnetic particles in magnetic fluid film
}

\author{
H.E. Hornga,*, S.Y. Yang ${ }^{\mathrm{a}, 1}$, Chun-Hui Chen ${ }^{\mathrm{a}}$, Y.P. Chiu ${ }^{\mathrm{a}}$, J.A. Chen ${ }^{\mathrm{b}}$, \\ Chin-Yih Hong ${ }^{\mathrm{b}}$, H.C. Yang ${ }^{\mathrm{c}}$ \\ ${ }^{a}$ Department of Physics, National Taiwan Normal University, Taipei 116, Taiwan \\ ${ }^{\mathrm{b}}$ Department of Mechanical Engineering, Da-Yeh University, Chang-Hwa 515, Taiwan \\ ${ }^{\mathrm{c} D e p a r t m e n t}$ of Physics, National Taiwan University, Taipei 106, Taiwan
}

\begin{abstract}
In this work, the influence of the sweep rates of applied magnetic fields on the agglomeration of magnetic particles in the magnetic fluid film are reported. It was observed that a smaller column agglomerated by magnetic particles results at a higher sweep rate as compared with that at a lower sweep rate. Besides, the magnetic moment per volume inside a column decreases rapidly when the sweep rate is raised from 0 to $15 \mathrm{Oe} / \mathrm{s}$, and almost remains constant as the sweep rate exceeds $20 \mathrm{Oe} / \mathrm{s}$. This variation implies that the packing of magnetic particles inside a column depends on the sweep rate. Thus, the sweep rate is a crucial variable determining the internal structure of columns. (C) 2001 Elsevier Science B.V. All rights reserved.
\end{abstract}

Keywords: Agglomeration; Magnetic fluids; Magnetic moment

\section{Introduction}

Because of its rich phenomena, the field-induced agglomeration of ferrite particles in magnetic fluid films under external magnetic fields has attracted much attention [1-3]. For example, the size of an agglomerated column at a given field strength was found to be linear with fluid concentration [1]. Jones and Belfield [2] described the magnetic field-dependent agglomeration of ferrite particles. Taketomi et al. [3] gave a thermodynamical instability theory about the ferrite particles' dispersion when agglomeration occurs. It was pointed out that magnetic interaction and thermal energy dominate the agglomeration of ferrite particles. Although researchers have examined the field-induced agglomer-

\footnotetext{
* Correspondence address: Department of Physics, National Taiwan Normal University, 88, Sec. 4, Ting-Chou Rd., Taipei 106, Taiwan. Fax: + 886-2-29309439.

${ }^{1}$ Present address: Institute of Physics, Academia Sinica, Nankang, Taipei 115, Taiwan

E-mail address: phyfv001@scc.ntnu.edu.tw (H.E. Horng).
}

ation of ferrite particles in magnetic fluids, the influence of the sweep rate of the applied magnetic field on this agglomeration is unclear. To understand the role of the sweep rate in the agglomeration of ferrite particles, we studied the behavior of the magnetic fluid film under external magnetic fields by applying the fields at various sweep rates.

\section{Experimental details}

The preparation of kerosene-based $\mathrm{MnFe}_{2} \mathrm{O}_{4}$ magnetic fluid with a saturated magnetization of $12.5 \mathrm{emu} / \mathrm{g}$ has been reported elsewhere [4]. The magnetic fluid thin film was obtained by injecting the magnetic fluid into a $6 \mu \mathrm{m}$-thick glass cell. To investigate the behavior of magnetic fluid film under external magnetic fields, the film was then inserted into a pair of solenoids, which provided a magnetic field perpendicular to the plane of the film. The desired magnetic field strength was achieved by using a fixed sweep rate to increase the field from zero to the desired strength $H$. The image of the structure formed in the magnetic fluid film was taken with a CCD 
when the structure reached equilibrium. With these images, the average diameter of the cross section of the columns and the separation between two columns under a given $H$ can be measured.

\section{Results and discussion}

By applying an external magnetic field at a given sweep rate $\mathrm{d} H / \mathrm{d} t$, say $2 \mathrm{Oe} / \mathrm{s}$, from zero to a final strength $H$, perpendicular to the plane of the magnetic fluid film, ferrite particles agglomerated to form magnetic columns. When the final $H$ exceeds a critical strength $(\sim 80$ Oe for the current sample), a hexagonal structure constructed by these columns is formed. Each column splits under higher $H$ 's $(>180 \mathrm{Oe}$ for $2 \mathrm{Oe} / \mathrm{s})$. At the hexagonal structure state, the averaged diameter $a$ of the columns in the film was found to be unchanged with variation of $H$, as shown in the inset of Fig. 1. However, $a$ becomes smaller when the columns split under higher $H$ s. As the $\mathrm{d} H / \mathrm{d} t$ is raised, the $a-H$ curve moves toward the region with smaller $a$ 's. The sweep rate dependent $a$ is shown in Fig. 1 for 100 Oe. It was found that $a$ decreases from 1.75 to $1.39 \mu \mathrm{m}$ when the $\mathrm{d} H / \mathrm{d} t$ increased from 2 to $40 \mathrm{Oe} / \mathrm{s}$. The smaller $a$ indicates that less particles are inside a column. Thus, the magnetic moment per column is reduced at a higher $\mathrm{d} H / \mathrm{d} t$.

According to our analogy study [5,6], the magnetic moment per column can be evaluated by fitting the experimental magnetic field-dependent column distance

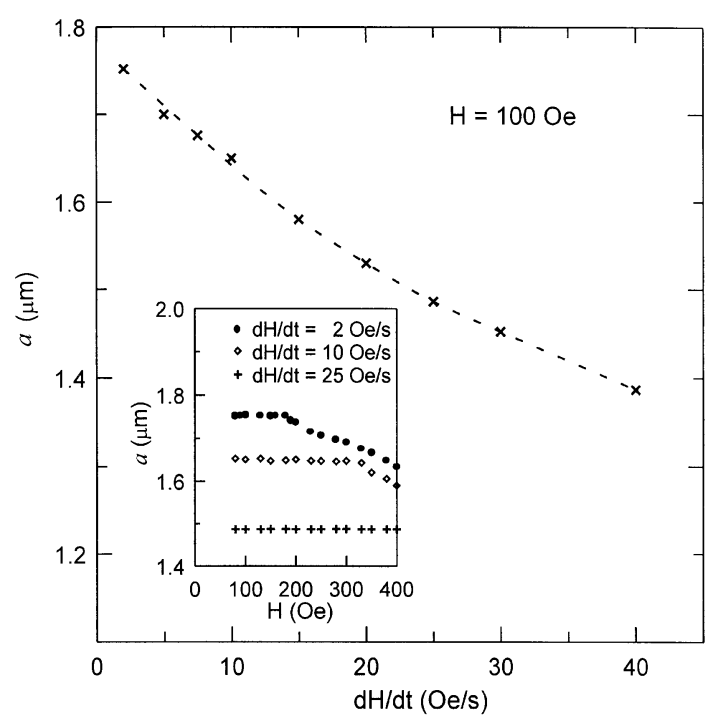

Fig. 1. The averaged diameter $a$ of the cross section of the columns versus the sweep rate $\mathrm{d} H / \mathrm{d} t$ of the applied magnetic field. The inset is the magnetic dependent column diameter $a-H$ curves at various $\mathrm{d} H / \mathrm{d} t$ 's.

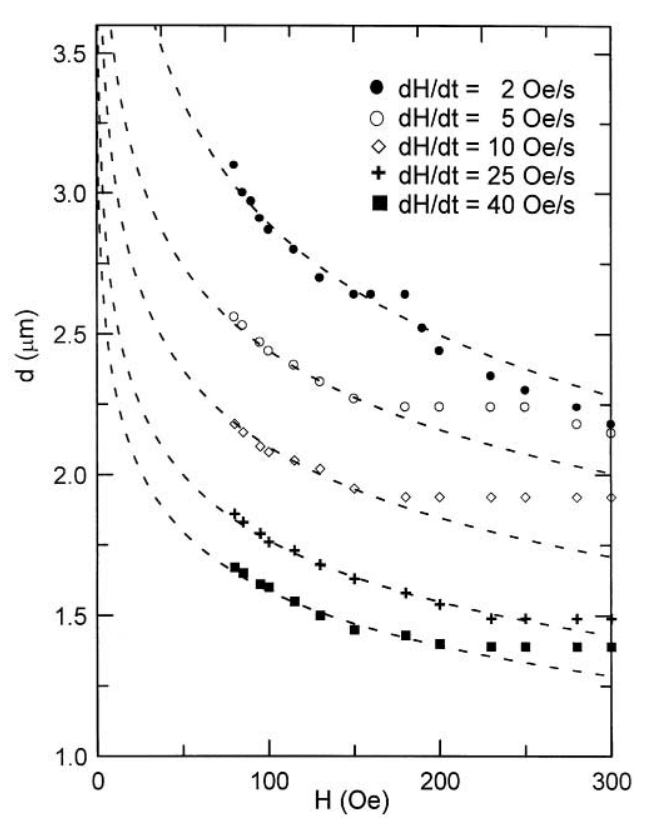

Fig. 2. The separation $d$ between two columns as a function of applied magnetic field $H$. The dashed lines denote the theoretical curves for various $\mathrm{d} H / \mathrm{d} t$.

Table 1

The averaged diameter $a$ 's of columns and the fitted magnetic moment $M_{\text {eff }}$ 's per column for various $\mathrm{d} H / \mathrm{d} t$ 's

\begin{tabular}{llllllllll}
\hline $\begin{array}{l}\mathrm{d} H / \mathrm{d} t \\
(\mathrm{Oe} / \mathrm{s})\end{array}$ & 2 & 5 & 7.5 & 10 & 15 & 20 & 25 & 30 & 40 \\
\hline$a(\mu \mathrm{m})$ & 1.75 & 1.70 & 1.68 & 1.65 & 1.58 & 1.53 & 1.49 & 1.45 & 1.39 \\
$M_{\text {eff }}$ & 2.99 & 2.70 & 2.17 & 1.57 & 1.28 & 1.19 & 1.05 & 0.99 & 0.91 \\
$\left(10^{-11} \mathrm{emu}\right)$ & & & & & & & & & \\
\hline
\end{tabular}

between two nearest columns ( $d-H$ curve) at the hexagonal structure state to the theoretical calculation. To do this, the $d-H$ curves at various $\mathrm{d} H / \mathrm{d} t$ 's were measured as shown in Fig. 2. A detailed discussion of the behavior of the $d-H$ curve at a certain $\mathrm{d} H / \mathrm{d} t$ has been introduced in our previous work [6]. On the other hand, for the theoretical consideration [6], the ordered hexagonal structure can be treated as a two-dimensional array of finite-size magnetic columns. The dipole interaction between columns and the interplay between the columns and $H$ govern the $d-H$ behavior which is then calculated as $H=M_{\text {eff }} f(a, L, d)$, where $M_{\text {eff }}$ is the magnetic moment per column, $L$ is the film thickness and $f$ is a function of $a, L$ and $d$, described in previous work [6]. The equation with the fitting parameter of $M_{\text {eff }}$ is applied to fit the experimental $d(H)$ data under lower $H$ 's and denoted by the dashed line in Fig. 2. A strong consistency between 


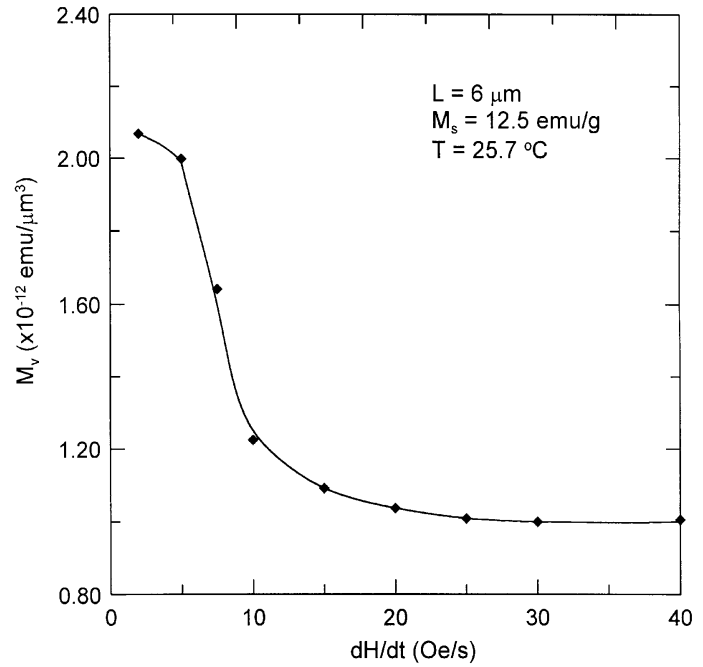

Fig. 3. The magnetic moment per volume $M_{\mathrm{v}}$ inside a column versus the sweep rate $\mathrm{d} H / \mathrm{d} t$.

the results of the experimental data and the corresponding theoretical curve is obtained. Through the fitting process, the magnetic moments at various $\mathrm{d} H / \mathrm{d} t$ 's are determined and listed in Table 1. The smaller column exhibits a lower magnetic moment.

With the $M_{\text {eff }}$, the magnetic moment per volume inside a column $M_{\mathrm{v}}$ can be found via $M_{\mathrm{v}}=M_{\text {ef } \mathrm{f}} /\left(\pi a^{2} L / 4\right)$. The $M_{\mathrm{v}}$ as a function of $\mathrm{d} H / \mathrm{d} t$ is shown in Fig. 3. The $M_{\mathrm{v}}$ reduced rapidly when the sweep rate was raised from 0 to $15 \mathrm{Oe} / \mathrm{s}$ and then almost remained constant as the sweep rate was raised higher than $20 \mathrm{Oe} / \mathrm{s}$. This variation in the $M_{\mathrm{v}}$ with the $\mathrm{d} H / \mathrm{d} t$ implies that the packing of ferrite particles inside a column is closer for lower $\mathrm{d} H / \mathrm{d} t$. Since the packing of magnetic particles inside a column is related to the internal structure of the column, the results shown in Fig. 3 imply that the sweep rate is very crucial to the internal structure of a column.

\section{Conclusion}

The size of the column agglomerated by ferrite particles becomes larger at a lower $\mathrm{d} H / \mathrm{d} t$. Also, experimental results reveal that the packing of ferrite particles inside a column is closer for a lower $\mathrm{d} H / \mathrm{d} t$.

\section{Acknowledgements}

This work was supported by the National Science Council of ROC. under grand No. NSC89-2112-M-003013.

\section{References}

[1] N.A. Yusuf, J. Phys. D 22 (1989) 1916.

[2] G.A. Jones, D.G. Belfield, J. Magn. Magn. Mater. 85 (1990) 37.

[3] S. Taketomi, H. Takahashi, N. Inaba, H. Miyajima, J. Phys. Soc. Japan 60 (1991) 1689.

[4] H.E. Horng, Chin-Yih Hong, W.B. Yeung, H.C. Yang, Appl. Opt. 37 (1998) 2674.

[5] Chin-Yih Hong, H.E. Horng, F.C. Kuo, S.Y. Yang, H.C. Yang, J.M. Wu, Appl. Phys. Lett. 75 (1999) 2196.

[6] S.Y. Yang, I.J. Jang, H.E. Horng, Chin-Yih Hong, H.C. Yang, Magn. Gidrodin. 36(1) (2000) 19. 